\title{
Optical Measurements of Presynaptic Release in Mutant Zebrafish Lacking Postsynaptic Receptors
}

\author{
Weiyan Li, Fumihito Ono, and Paul Brehm \\ Department of Neurobiology and Behavior, State University of New York at Stony Brook, Stony Brook, New York 11794
}

Differentiation of presynaptic nerve terminals is mediated, in part, through contact with the appropriate postsynaptic target cell. In particular, studies using dissociated nerve and muscle derived from Xenopus embryos have indicated that the properties of transmitter release from motor neurons are altered after contact with skeletal muscle. This maturation of presynaptic function has further been linked to retrograde signaling from muscle that involves activation of postsynaptic ACh receptors. Using FM1-43 optical determinants of exocytosis, we now compare calcium-mediated exocytosis at neuromuscular junctions of wild-type zebrafish to mutant fish lacking postsynaptic ACh receptors. In response to either high-potassium depolarization or direct electrical stimulation, we observed no differences in the rate or extent of FM1-43 destaining. These data indicate that the acquisition of stimulus-evoked exocytosis at early developmental stages occurs independent of both postsynaptic receptor and synaptic responses in zebrafish.

Key words: zebrafish; mutant; neuromuscular junction; acetylcholine receptor; motor neuron; presynaptic differentiation; exocytosis

\section{Introduction}

Presynaptic and postsynaptic development at the neuromuscular junction are mutually inductive events, and much interest surrounds identification of the signaling pathways involved. For example, presynaptically derived agrin serves as the trigger for the localization of receptors and the associated postsynaptic machinery in muscle cells (for review, see Sanes and Lichtman, 2001). Involvement of postsynaptic retrograde signals in fostering presynaptic differentiation has also been shown to occur at vertebrate nerve-muscle synapses. In rapsyn null mice, formation of postsynaptic receptor clusters and presynaptic terminals is compromised (Gautam et al., 1995; Nguyen et al., 2000). Postsynaptic modulation of transmitter release machinery has been provided from studies that use Xenopus nerve and muscle wherein the formation of synaptic contacts can be strictly controlled. Measurement of synaptic responses from myocytes shows that both motor neuron somas and growth cones can spontaneously release ACh before contact with muscle (Kidokoro and Yeh, 1982; Hume et al., 1983; Young and Poo, 1983; Chow and Poo, 1985). However, contact with muscle potentiates transmitter release, as reflected in the increased size (Evers et al., 1989; Liou et al., 1997, 1999) and frequency of spontaneous events (Xie and Poo, 1986; Evers et al., 1989). Additionally, the amplitude of the stimulusevoked postsynaptic response is increased, indicating that the retrograde signaling by muscle also affects calcium-mediated exocytosis in the presynaptic terminal (Sun and Poo, 1987).

The retrograde signals that purportedly mediate presynaptic

Received Aug. 13, 2003; revised Sept. 12, 2003; accepted Sept. 22, 2003.

This research was supported by National Institutes of Health Grant NS18205 to P.B. We thank the University of Oregon zebrafish facility for providing the mutant line nic-1. We also thank Dr. Yoshiaki Kidokoro for the suggestion of using FM1-43.

Correspondence should be addressed to Paul Brehm, Department of Neurobiology and Behavior, State University of New York at Stony Brook, Stony Brook, NY 11794. E-mail: pbrehm@notes.cc.sunysb.edu.

Copyright $\odot 2003$ Society for Neuroscience $\quad 0270-6474 / 03 / 2310467-08 \$ 15.00 / 0$ potentiation include neurotrophins 3 (Liou and Fu, 1997; Liou et al., 1997) and 4 (Liou et al., 1997; Wang and Poo, 1997). Most significantly, presynaptic potentiation is inhibited after transient blockade of the postsynaptic ACh receptors with D-tubocurarine (Liou and Fu, 1997; Liou et al., 1997, 1999; Wan and Poo, 1999). These provocative findings have led to the suggestion that ACh receptor-mediated synaptic activity is directly involved in mediating presynaptic transmitter release mechanisms. This idea receives additional support from studies on invertebrate synapses. In Caenorhabditis elegans, induction of the presynaptic vesicle release machinery is triggered through release of a postsynaptic signaling peptide (Doi and Iwasaki, 2002). Also, in the mollusc Helisoma, acquisition of excitation-secretion machinery at nerve-muscle synapses involves activity-dependent signaling through the postsynaptic receptors (Poyer and Zoran, 1996). Finally, at Drosophila neuromuscular synapses, the postsynaptic glutamate receptor regulates quantal size and quantal content of developing presynaptic terminals (DiAntonio et al., 1999).

Although all of the above experiments point to the importance of retrograde signals in presynaptic events, direct tests for a role of postsynaptic receptors in altering developmental acquisition of presynaptic release machinery have been hampered by the lack of ACh receptor (AChR) null animal model systems. In mammals, total ACh receptor knock-outs have not been published because of the lethal consequences at early developmental times. We now use two different mutant lines of paralytic zebrafish that lack muscle nicotinic AChRs. Unlike the case for mammals, these receptorless fish are able to survive long enough to establish well developed neuromuscular junctions (Westerfield et al., 1990; Ono et al., 2001). Using FM1-43 destaining as a measure of presynaptic vesicular release, we have been able to assess presynaptic exocytosis in the absence of receptors, the functional postsynaptic response element. Our findings indicate that, unexpectedly, the 
presynaptic vesicular release in the mutant fish lacking receptors was comparable with that in wild-type fish.

\section{Materials and Methods}

Wild-type, sofa potato, and relaxed (Granato et al., 1996) mutant zebrafish were obtained from The Max Planck Institute (Tübingen, Germany) and nic- 1 fish were obtained from the University of Oregon (Eugene, OR) (Westerfield et al., 1990). Homozygous nic-1 (Westerfield et al., 1990), sofa potato (Ono et al., 2001), and relaxed (Ono et al., 2001) mutant embryos and larva were identified on the basis of an inability to move when prodded with a fine-tip needle. These homozygotes generally died within $7 \mathrm{~d}$ after fertilization because of complications resulting from the paralysis. Therefore, most experiments were performed on fish larvae at 4-5 d after fertilization. (Please note that according to our method of aging, a fish is considered to be $1 \mathrm{~d}$ old between 24 and $48 \mathrm{hr}$ after fertilization.) At day 2, the mutant fish were dechorionated to facilitate additional development. All fish were maintained at $28^{\circ} \mathrm{C}$ in HBSS containing the following (in $\mathrm{mM}$ ): $137 \mathrm{NaCl}, 5.4 \mathrm{KCl}, 1 \mathrm{MgSO}_{4}, 0.44$ $\mathrm{KH}_{2} \mathrm{PO}_{4}, 0.25 \mathrm{Na}_{2} \mathrm{HPO}_{4}, 4.2 \mathrm{NaHCO}_{3}, 1.3 \mathrm{CaCl}_{2}$, and $5 \mathrm{Na}-\mathrm{HEPES}$ diluted to $10 \%$ strength.

FM1-43 labeling. Before experimentation, 4- to 5-d-old fish larvae were anesthetized in full-strength HBSS containing 0.02\% MS222 (Sigma, St. Louis, MO). The immobile fish were then pinned to a Sylgard (Dow Corning, Midland, MI)-coated dish both at head and extreme tail end using electrolytically sharpened tungsten needles. The skin was then gently peeled away from the exposed side of the fish to permit access to FM1-43 (Molecular Probes, Eugene, OR) and $\alpha$-bungarotoxin ( $\alpha$-Btx) (Calbiochem, La Jolla, CA). The fish were first treated with $0.1 \mu \mathrm{M} \alpha$-Btx for $15 \mathrm{~min}$ to block synaptic transmission. In some experiments, $0.1 \mu \mathrm{M}$ Alexa Fluor 594-conjugated $\alpha$-Btx (Molecular Probes) was used to fluorescently label the AChRs. Alexa 594 was chosen to label muscle AChRs because the overlap of its excitation spectrum with that of FM1-43 is minimal, thus avoiding possible optical spillover between the two dyes.

Fish were then transferred to an HBSS containing $10 \mu \mathrm{M}$ FM1-43 to allow preloading penetration of the dye molecules into the interstitial regions of the tail. After $15 \mathrm{~min}$, this solution was replaced by a highpotassium loading solution composed of the following (in $\mathrm{mM}$ ): $97 \mathrm{NaCl}$, $45 \mathrm{KCl}, 1 \mathrm{MgSO}_{4}, 5 \mathrm{Na}-\mathrm{HEPES}$, and $5 \mathrm{CaCl}_{2}$, containing $10 \mu \mathrm{M} \mathrm{FM1-43.}$ After $5 \mathrm{~min}$, the fish were transferred to HBSS containing $10 \mu \mathrm{M}$ FM1-43 for an additional $3 \mathrm{~min}$, after which loading was complete. The fish were subsequently washed three times with a low-Ca HBSS $\left(0.5 \mathrm{~mm} \mathrm{CaCl}_{2}\right)$ in an attempt to minimize spontaneous release of loaded synaptic vesicles. To facilitate the removal of residual FM1-43 from the interstitial spaces, fish were transferred into a low-Ca HBSS solution containing $1 \mathrm{~mm}$ Advasep 7 (CyDex, Overland Park, KS) for 5 min (Kay et al., 1999). Finally, the fish were transferred through six consecutive 3 min washes in Advasep 7-free low-Ca HBSS.

Confocal microscopy. With the skinless side facing down, the fish were placed on a coverslip and buried in 1\% agarose dissolved in HBSS. Once solidified, this agarose block was inverted and placed on the stage of a Zeiss (Thornwood, NY) FS-motorized Axioscope. The superficially located terminals that were labeled with FM1-43 were then imaged using a Yokogawa confocal spinning disk unit (Solamere Technology, Salt Lake City, UT). This method of confocal analysis offered a much faster acquisition rate compared with traditional scanning confocal methods, thus minimizing problems associated with photobleaching and phototoxic damage to the tissue. To completely eliminate the problem of photobleaching, we acquired images at long intervals, every 3 sec. FM1-43 fluorescence was excited at $488 \mathrm{~nm}$ using an argon laser and long-pass filtered at $520 \mathrm{~nm}$.

For experiments involving simultaneous labeling of AChRs and FM143, a Zeiss LSM 510 scanning confocal inverted microscope equipped with the Meta system was used. AChRs were labeled using Alexa 594 $\alpha$-Btx. Alexa 594-associated fluorescence was excited at $633 \mathrm{~nm}$ and long-pass filtered at $650 \mathrm{~nm}$. FM1-43-associated fluorescence was excited at $488 \mathrm{~nm}$ and bandpass filtered between 505 and $530 \mathrm{~nm}$. In some experiments, we simultaneously imaged motor neuron terminals labeled with green fluorescent protein (GFP) and FM1-43. For this purpose, we generated heterozygote lines of nic-1 and sofa potato that constitutively expressed GFP in motor neurons under the control of the fish Islet 1 promoter. This promotor drove expression of cytoplasmic GFP in the secondary motor neurons. Simultaneous imaging of GFP and FM1-43 fluorescence was provided using $488 \mathrm{~nm}$ excitation and $510-595.6 \mathrm{~nm}$ emission spectrum. In these experiments, the mounting of the fish had to be modified to accommodate the use of the inverted microscope. For this purpose, the fish were mounted in a $1 \%$ agarose block with the intact skin side facing down. The transparency of the skin permitted confocal viewing of neuromuscular synapses by the inverted microscope.

FM1-43 destaining. Once loaded with FM1-43, two different methods of nerve terminal depolarization were used to destain. The first used high-potassium HBSS, wherein $45 \mathrm{~mm} \mathrm{~K}^{+}$was substituted for $\mathrm{Na}^{+}$. This high-potassium HBSS was directly applied via a pressure-driven pipette. Alternatively, the nerve terminals were directly depolarized by means of a local electrical field. For this purpose, two platinum-iridium wires (0.005 inches in diameter), $\sim 400 \mu \mathrm{m}$ apart, were gently pressed onto the fish so that they flanked the area of interest. Both wires were insulated with melted glass along their length so that only the tips were exposed to the solution. A Grass stimulator (Grass-Telefactor, West Warwick, RI) was used to deliver $10 \mathrm{~Hz}$ trains of $3 \mathrm{~V}, 2$ msec pulses. Recordings of evoked synaptic current from muscle have indicated that these stimulus parameters result in consistent stimulation of motor neurons (Ono et al., 2002). Typically, the train lasted 3-5 min to achieve maximal destaining of nerve terminals.

Image processing and data analysis. The size of individually labeled boutons was determined by outlining the border with an ellipse. The short and long axes were measured, and the mean of the two values was used as the diameter to compute overall area of the bouton. Because the images are fuzzy at high gain, our blind measurements on wild-type and mutant fish ensured that the measurements were made in the same way on all fish.

To quantitate FM1-43 destaining with the spinning disk confocal system, real-time and time-lapse images were captured using a Stanford Photonics (Palo Alto, CA) XR Mega-10 intensified CCD camera. For this purpose, the average of eight $1024 \times 1024$ real-time images were acquired at $3 \mathrm{sec}$ intervals. The quantitative analyses of fluorescence intensity and distribution were performed on each average image using QED Camera Plugin software (QED Imaging, Pittsburgh, PA). Between two and five brightly labeled varicosities were selected from each average image for measurement. The average change in pixel intensity was determined for each varicosity as a function of time by subtracting the average background fluorescence. The shape of terminals was assumed to remain unchanged during the stimulation. Statistical analysis was performed using Student's independent $t$ tests in which significance was set at $p<$ 0.05 . To analyze dual-wavelength fluorescence associated with FM1-43 and Alexa 594, scanning confocal microscopic images were recorded and analyzed with Zeiss LSM 510 software. The FM1-43 and GFP fluorescence signals were separated using LSM 510 Meta software.

\section{Results}

The muscles in the tails of zebrafish larva are segmentally organized with innervation occurring both at the ends as well as the midsection (Fig. 1 $A, B$ ). When labeled with FM1-43, the fluorescence was seen at locations on the muscle surface corresponding to these two regions (Figs. 1, 2). Most often, the most intense fluorescence labeling corresponded to individual bright varicosities (Figs. 1C, 2). Processes connecting these varicosities were weakly labeled and could occasionally be traced back to cell bodies in the spinal cord. Labeling of the spinal cord neurons by FM1-43 resulted in an elevated level of background fluorescence in the dorsal part of the fish, thus restricting analysis to the synapses on ventral muscle cells. Even after extensive washing in HBSS, the muscle cells exhibited diffuse membrane fluorescence. However, the inclusion of Advasep 7 in the washing solution decreased nonspecific muscle fluorescence to levels that were barely detectable. Additionally, Advasep 7 did not result in any 

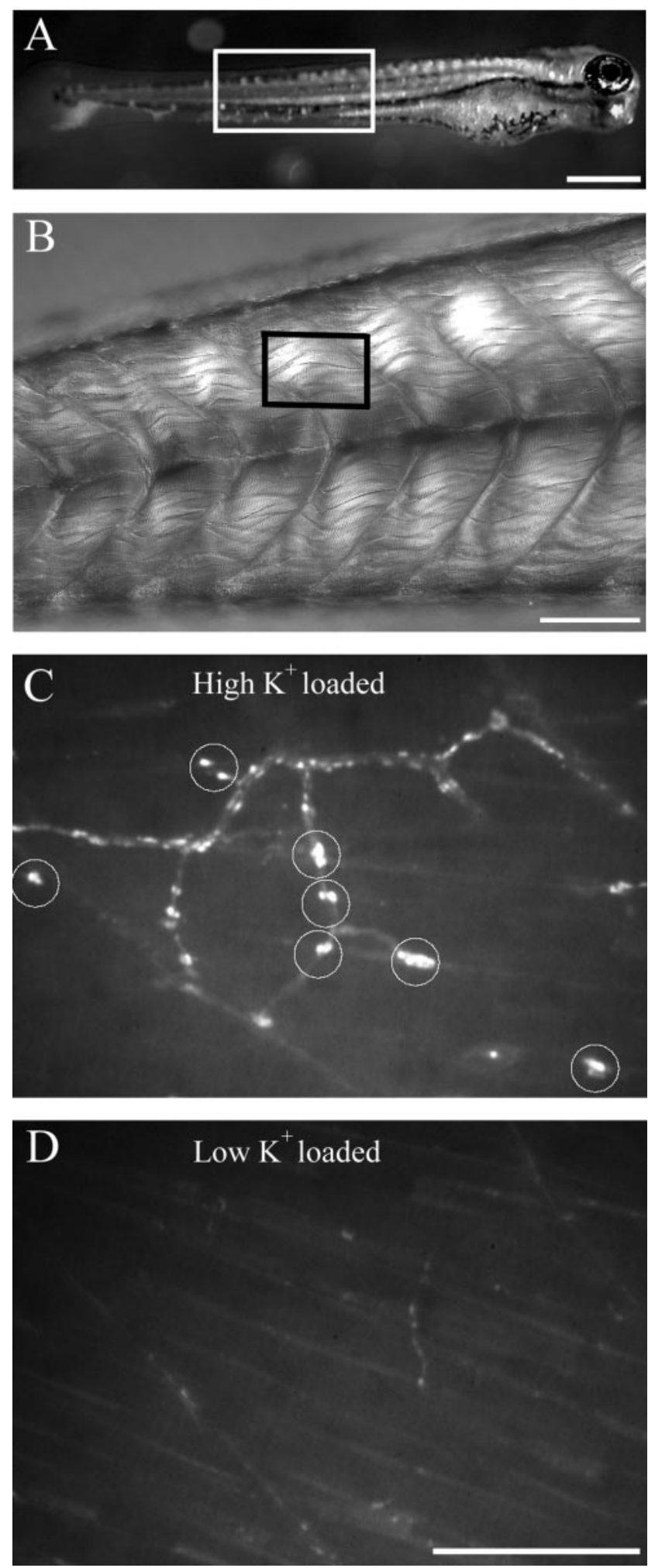

Figure 1. FM1-43 labeling of nerve terminals is activity dependent. $A$, Low-power photograph of a 5-d-old larva. The region of tail corresponding to the photograph in $B$ is outlined. $B$, A differential interference contrast photograph corresponding to the region of tail muscle outlined in $A$ showing the individual muscle cells and myocomata. The outlined region in $B$ corresponds approximately to the size of the regions shown in C and D. C, High-power fluorescence photograph of the tail from a fish that was loaded with $10 \mu \mathrm{M}$ FM1-43 in the presence of a high-potassium HBSS. The circles indicate varicosities that were dye loaded. D, High-power fluorescence photograph of the tail of a fish different from that shown in C that was loaded with $10 \mu \mathrm{MFM} 1-43$ in low-potassium HBSS. The areas shown in both C and $D$ correspond to approximately one tail segment. Scale bars: $A, 500 \mu \mathrm{m} ; B, 100 \mu \mathrm{m} ; C, D, 20 \mu \mathrm{m}$. obvious decrease in the fluorescence associated with nerve terminals.

Several lines of evidence from wild-type fish indicate that the majority of varicosities labeled by FM1-43 represented presynaptic terminals containing labeled vesicles. The first line of evidence for FM1-43 labeling of synaptic terminals comes from comparison of high-potassium versus low-potassium loading conditions. High-potassium loading solution consistently resulted in effective FM1-43 labeling of varicosities (Fig. 1C), whereas use of a low-potassium solution (normal HBSS) resulted in weakly labeled varicosities (Fig. $1 D$ ). A second line of evidence comes from labeling of postsynaptic ACh receptors. In wild-type fish, the brightly labeled FM1-43 varicosities colocalized with postsynaptic clusters of AChRs labeled by Alexa 594-conjugated $\alpha$-Btx (Fig. 2A). A third line of evidence comes from analysis of transgenic fish expressing GFP in the secondary motor neurons that show colocalization of the GFP and FM1-43 label (Fig. 3). Occasionally, we observed FM1-43-labeled varicosities that failed to exhibit GFP label because the Islet1 promoter, used to drive GFP expression, is specific to a subset of secondary motor neurons. Additionally, there were occasional sites of GFP label that were not associated with FM1-43 label, because of GFP filling of axons in addition to the terminals. The final evidence that FM1-43 labels terminals comes from the observation that labeled varicosities were effectively destained in response to application of high potassium (Figs. 2, 4, 5) or by electrical field stimulation (Fig. 6). This loss of FM1-43 fluorescence at brightly labeled varicosities was not accompanied by a decrease in Alexa $594 \alpha$-Btx label (Fig. 2 B).

The labeling of wild-type fish by FM1-43 was subsequently compared with sofa potato and nic-1, two paralytic mutant zebrafish lines that lack postsynaptic AChRs (Westerfield et al., 1990; Ono et al., 2001). Mutant lines were both effectively loaded with FM1-43 by use of the same loading procedures established for wild-type fish. As seen in wild-type fish, the FM1-43 label colocalized to expressed GFP in the subset of secondary motor neurons of both nic-1 and sofa potato (Fig. 3). Additionally, no systematic difference in either the position or number of GFPand FM1-43-labeled varicosities was observed for wild type, nic-1, or sofa potato. There is variability in the ability of both GFP and FM1-43 to label the myocomatal ends of the muscle cells. The origin of this variability is not known, but staining with $\alpha$-Btx also reveals that superficial muscle cells tend to label on the ends, whereas deeper muscle layers fail to do so. To quantitatively compare loading of FM1-43 for mutant and wild-type fish, the average intensity and mean diameter for varicosities were compared under identical gain settings. The average diameter (mean of large and small axes) and fluorescence intensity, after background correction, were quantitated for each varicosity (Table 1). Varicosities from wild-type fish measured $1.52 \pm 0.39 \mu \mathrm{m}$ in diameter and $560 \pm 80$ arbitrary units (a.u.) in fluorescence intensity (50 varicosities from 11 fish). For nic-1, the varicosity diameter averaged at $1.51 \pm 0.34 \mu \mathrm{m}$ and the intensity averaged $537 \pm 100$ a.u. (45 varicosities from 11 fish). For sofa potato, the varicosity diameter averaged at $1.58 \pm 0.38 \mu \mathrm{m}$ and intensity averaged $560 \pm 97$ a.u. (55 varicosities from 12 fish). None of the values obtained for nic-1 or sofa potato were significantly different from those obtained for wild-type fish (Table 1).

To quantitate the rate and extent of exocytosis from presynaptic motor neuron terminals in wild-type fish, individually identified brightly labeled terminals were challenged with high potassium. Within $30 \mathrm{sec}$ of local application of high-potassium solution, the fluorescence associated with brightly labeled vari- 
cosities began to decrease rapidly, until plateau levels were achieved (Fig. 4). Because of a noisy signal and the slow speed of the high-potassium application, we were not able to compare the kinetics of destaining or the latency of onset of destaining in mutant and wild-type fish. However, for individual varicosities, we were able to determine the following: (1) the fractional fluorescence change $(\Delta F / F)$ during exposure to high-potassium solution and (2) the destaining time $\left(T_{10-90 \%}\right.$, time required for $80 \%$ of fluorescence change). In wild-type fish, the maximal destaining by high-potassium solution corresponded to $77 \pm 12 \%$ of fluorescence loss, and change of fluorescence corresponding to $10-90 \%$ of maximal destaining required $47 \pm 22 \mathrm{sec}$ (20 varicosities from six fish). The same destaining protocol was applied to the mutant lines nic-1 and sofa potato for comparison with wildtype fish. In both mutant lines, the synaptic varicosities labeled by FM1-43 were readily destainable by high-potassium stimulation (Fig. 5). Fractional loss of fluorescence $(\Delta F / F)$ for nic-1 averaged at $74 \pm 13 \%$, and $T_{10-90 \%}$ averaged at $38 \pm$ $16 \mathrm{sec}$ ( 18 varicosities from five fish). $\Delta F / F$ for sofa potato averaged at $81 \pm 8 \%$, and $T_{10-90 \%}$ averaged at $43 \pm 16 \mathrm{sec}$ (23 varicosities from six fish). None of the values obtained for wild type, nic-1, and sofa potato were significantly different.

Additional physiological tests of depolarization-induced destaining were provided by electrical stimulation of the motor neurons. For this purpose, identified FM1-43-loaded terminals in nic-1, sofa potato, and wild-type fish were stimulated using $10 \mathrm{~Hz}$ trains of brief depolarizations. In all fish studied, both mutant and wild type, a decrease in fluorescence intensity of synaptic varicosities occurred in response to electrical stimulation (Fig. 6). Additionally, unlike the response to high potassium, which requires diffusion and penetration, the onset of electrically evoked destaining was immediate. On the basis of the same analysis described previously, nic- 1 fish had an average $\Delta F / F$ value of $72 \pm 10 \%$ and an average $\mathrm{T}_{10-90 \%}$ value of $85 \pm 23 \mathrm{sec}$ ( 18 varicosities from five fish). Sofa potato $\Delta F / F$ averaged at $72 \pm$ $12 \%$, and $T_{10-90 \%}$ averaged at $80 \pm 30 \mathrm{sec}$ (21 varicosities from seven fish). These values were not significantly different from those obtained for wild-type fish $\left(\Delta F / F=78 \pm 10 \% ; T_{10-90 \%}=94 \pm 32 \mathrm{sec} ; 22\right.$ varicosities from six fish). Overall, the extent of destaining by electrical stimulation in all fish was similar to that obtained by high-potassium stimulation. However, the rate of destaining was almost twice as slow for electrical stimulation (Table 1). This difference is likely attrib-
FM 1-43
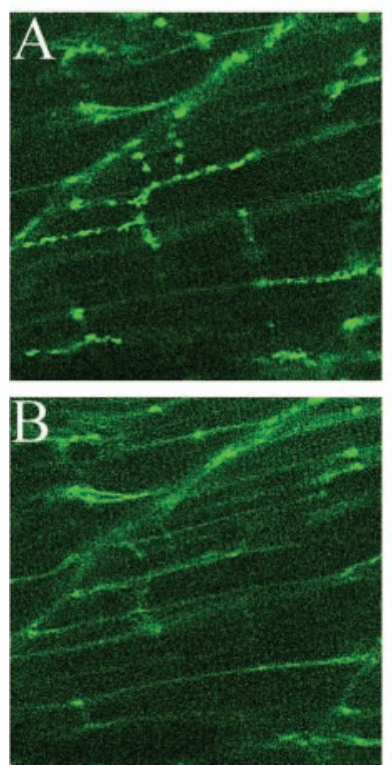

Alexa 594- $\alpha$-Btx
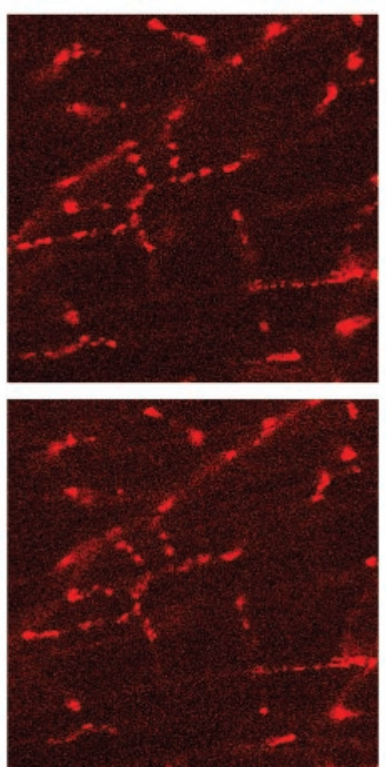

Merge
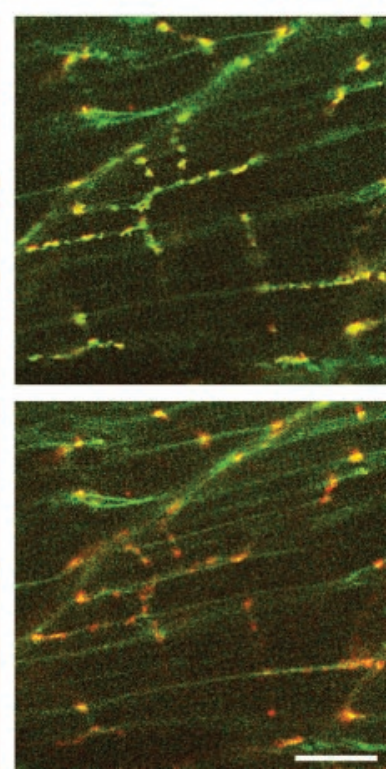

Figure 2. FM1-43 label colocalizes with AChR clusters in wild-type fish. A, A 5-d-old wild-type fish colabeled with $0.1 \mu \mathrm{M}$ Alexa 594-conjugated $\alpha$-Btx and $10 \mu \mathrm{m}$ FM1-43. The green FM1- 43 labeling of synaptic varicosities (left) and the red labeling of AChR clusters by Alexa $594 \alpha$-Btx (middle) colocalize in the merged image (right). B, The same field is shown after 3 min of high-potassium treatment. The area shown corresponds to approximately one tail segment. Scale bar, $20 \mu \mathrm{m}$.
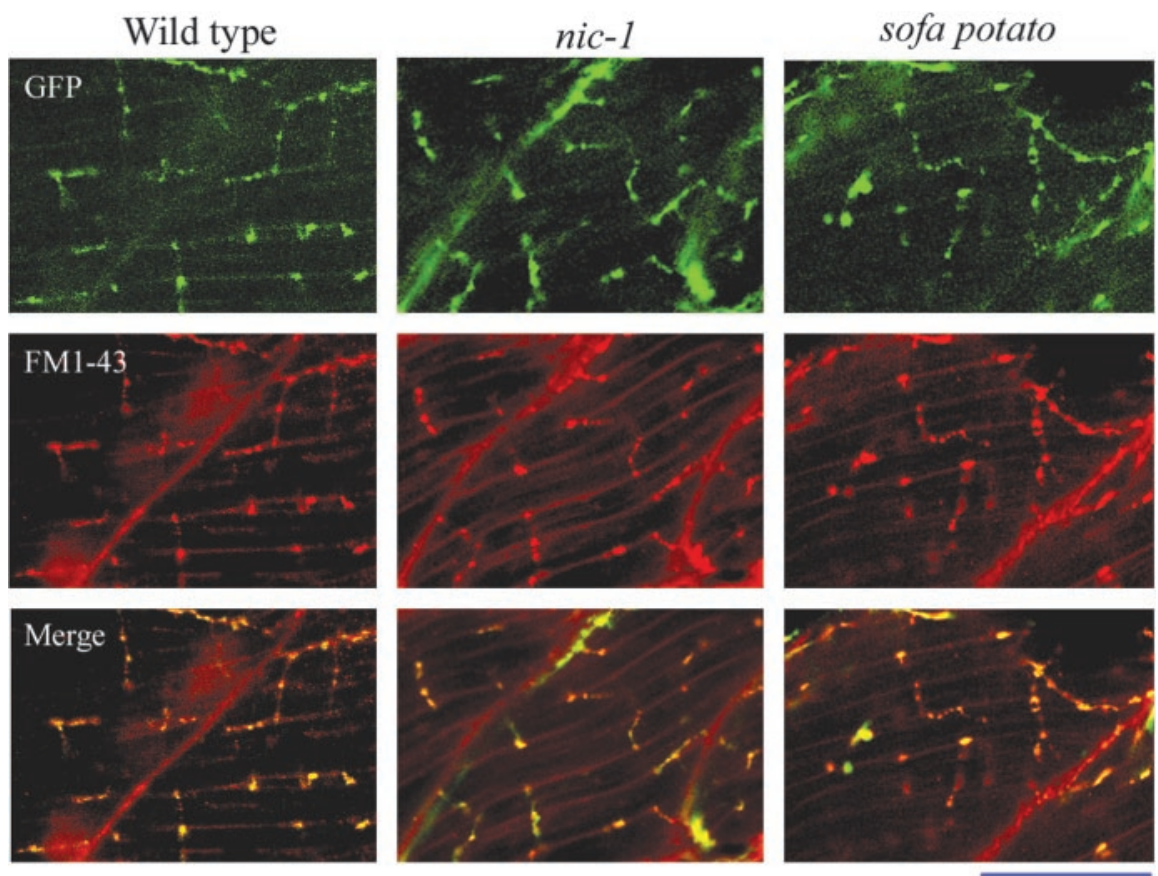

Figure 3. FM1-43 labels nerve in both wild-type and mutant synapses. Secondary motor neurons were visualized in transgenic wild-type and mutant lines of fish that were expressing cytoplasmic GFP. The GFP was constitutively expressed in the motor neurons of all three lines using a neuron-specific Islet1 promoter (top). Three Islet1 fish, corresponding to wild-type, nic-1, and sofa potato, were labeled with $10 \mu \mathrm{m}$ FM1- 43 (middle). Merged images indicate that the motor neuron GFP label colocalizes with the FM1- 43 label (bottom). Scale bar, $50 \mu \mathrm{m}$.

utable to the relative abilities of $10 \mathrm{~Hz}$ stimulation versus high potassium to provide maximal and maintained exocytosis.

One qualitative difference between mutant and wild-type fish was manifest in both the initial intensity of staining and the tendency to spontaneously destain over time. Typically, in the ab- 


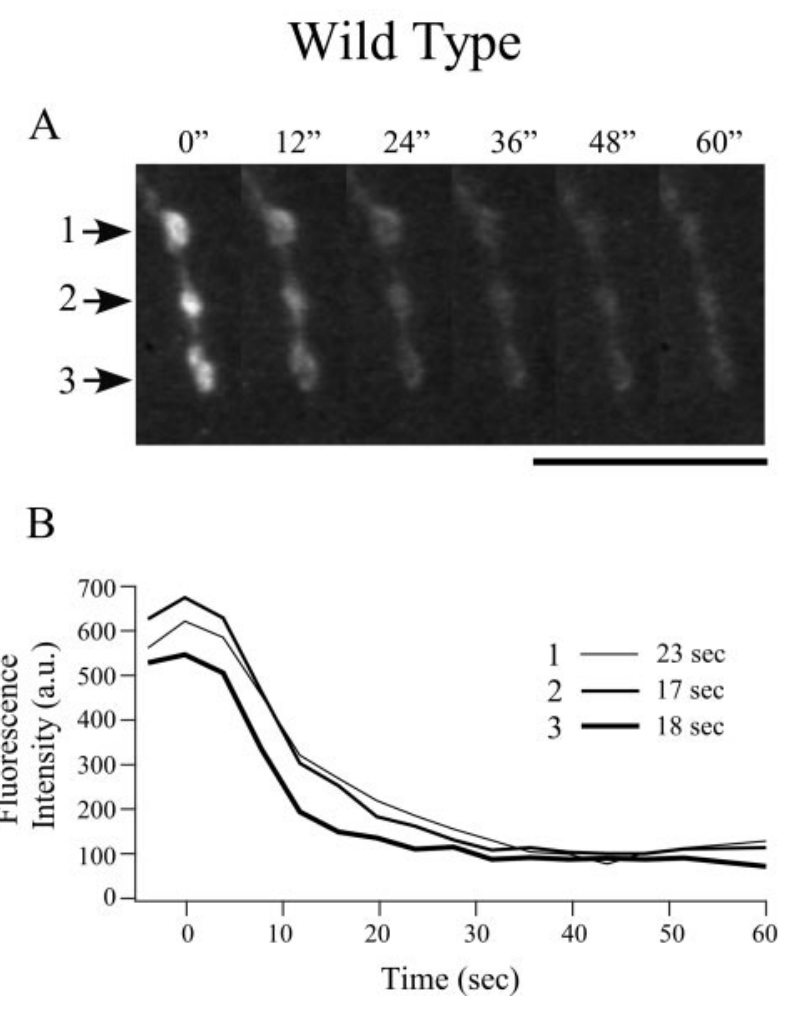

Figure 4. High-potassium destaining kinetics of FM1-43-loaded synaptic varicosities in wild-type fish. $A$, Time-lapse images of three varicosities (indicated by arrows 1-3) during high-potassium-induced destaining. Representative images are shown at 12 sec intervals after perfusion of high-potassium solution. Scale bar, $10 \mu \mathrm{m}$. B, Time-course plot of fluorescence loss after background correction for the three individual varicosities shown in $A$. Time 0 is determined by the slight twitch of muscles with arrival of high-potassium solution. Fluorescence intensity was measured every $4 \mathrm{sec}$. The time required for decay from 90 to $10 \%$ of peak fluorescence determined for each trace is indicated alongside the corresponding legend bar.

sence of either TTX or $\alpha$-Btx, the wild-type fish showed fewer brightly labeled varicosities than either nic-1 or sofa potato. This difference was not a consequence of photobleaching, because in control experiments, neither the background label nor the intensity of the occasional nondestainable nerve tracts decreased over the course of the experiment. Instead, it appears that the varicosities of wild-type fish exhibited a much greater tendency to spontaneously destain. The spontaneous destaining seen in wild-type fish was also not observed in another mutant fish, relaxed, which have normal synaptic transmission but are unable to contract because of defective excitation-contraction coupling (Ono et al., 2001). The spontaneous destaining in wild-type fish was blocked by treatment with either $0.1 \mu \mathrm{M} \alpha$-Btx or $1 \mu \mathrm{M}$ tetrodotoxin. Thus, to counter this tendency in wild-type fish, $\alpha$-Btx was routinely added to the preincubation solutions.

\section{Discussion}

Two mutant zebrafish lines lacking muscle nicotinic AChRs offered the opportunity to test directly whether postsynaptic receptors play a role in presynaptic function. Previous studies on both lines had indicated that the neuromuscular junctions were remarkably normal in appearance, despite the lack of postsynaptic receptors (Westerfield et al., 1990; Ono et al., 2001), but presynaptic function was not assessed in either study. The absence of muscle AChRs in the nic-1 line results from a point mutation in the $\alpha$-subunit of the receptor, precluding surface expression of this multisubunit receptor (Sepich et al., 1998). In sofa potato, the

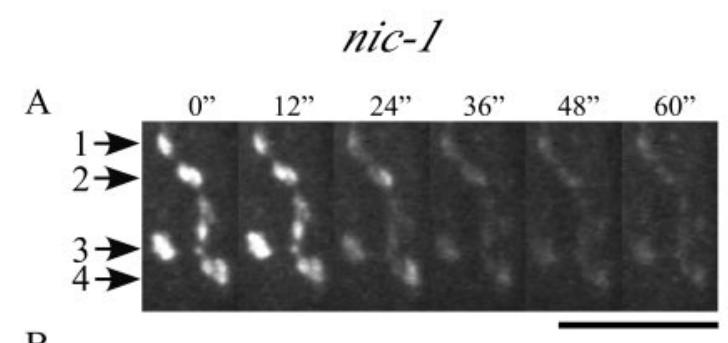

B
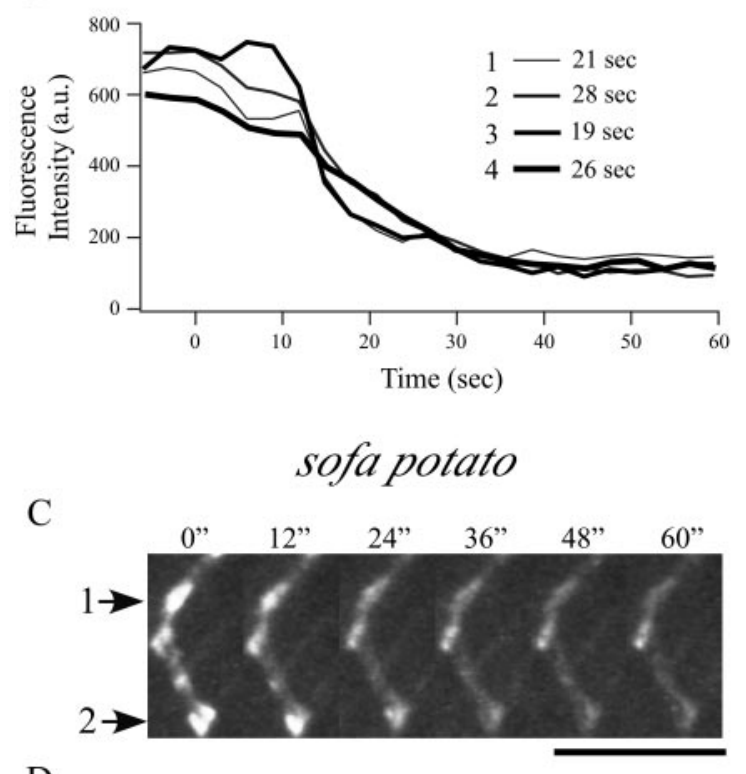

D

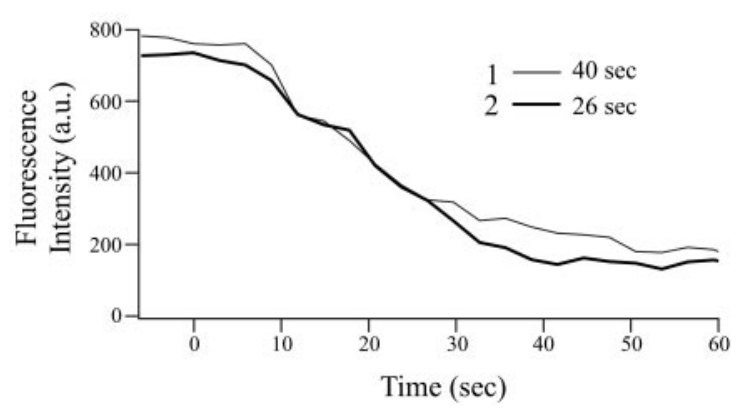

Figure 5. High-potassium destaining kinetics of FM1-43-loaded varicosities in mutant zebrafish. Representative time-lapse images of labeled varicosities (indicated by arrows) are shown at 12 sec intervals for nic- $1(A)$ and sofa potato $(C)$ after perfusion of high-potassium solution. Scale bars, $10 \mu \mathrm{m}$. The associated time course of fluorescence loss for each varicosity is plotted in $B$ and $D$ for each mutant after background correction. Time 0 is determined by the slight twitch of muscles with arrival of high-potassium solution. Fluorescence intensity was measured every $3 \mathrm{sec}$. The time required for decay from 90 to $10 \%$ of peak fluorescence determined for each trace is indicated alongside the corresponding legend bar.

locus underlying the loss of receptors has not yet been published. However, a complete lack of surface receptors in sofa potato was indicated by a lack of postsynaptic currents as well as by an absence of labeling by both $\alpha$-Btx and anti- $\alpha$-subunit antibodies (Ono et al., 2001). In mammals, ACh receptor knock-out is lethal at early stages of development, precluding the kind of analysis provided in this study by both nic-1 and sofa potato zebrafish. Because postsynaptic responses could no longer be used to assess presynaptic release in these mutant fish, we turned to the use of optical measurements of exocytosis. The accessibility of the motor nerve terminals facilitated the use of optical measurements developed for study of amphibian (Betz and Bewick, 1992, 1993), 
A
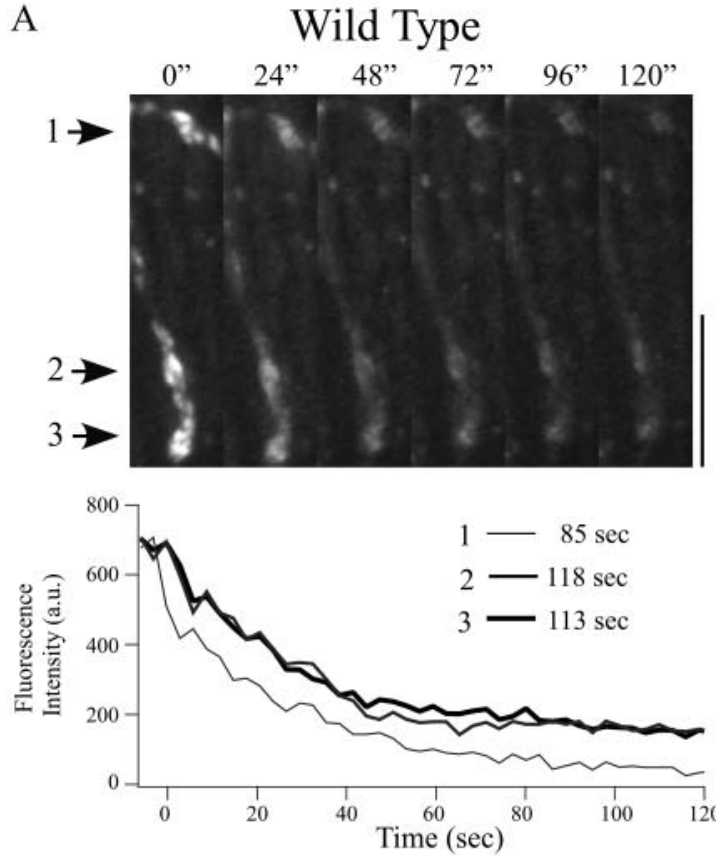

B
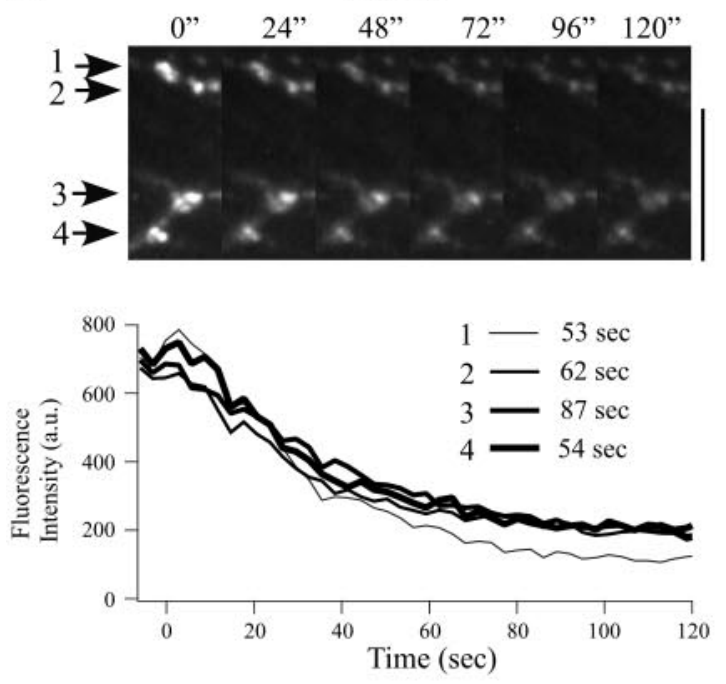

$\mathrm{C}$
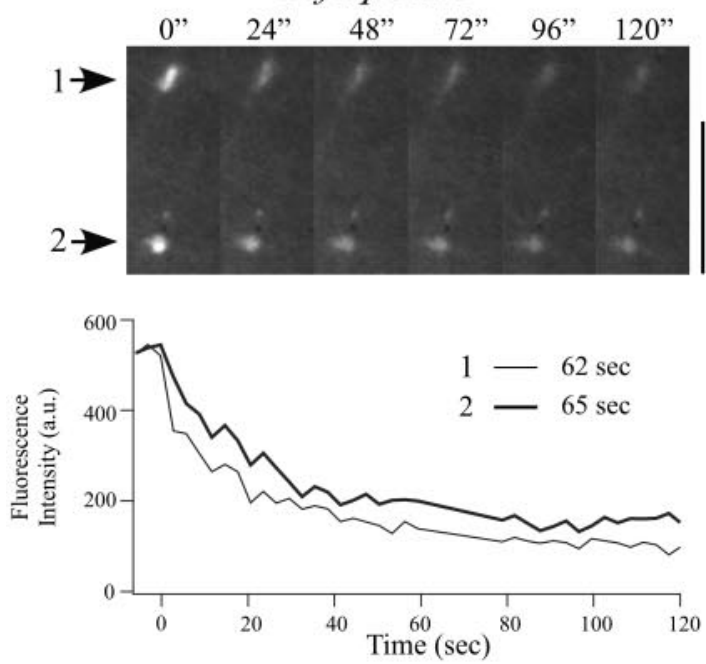

mammalian (Betz et al., 1992; Ribchester et al., 1994), and Drosophila nerve-muscle preparations (Kuromi and Kidokoro, 1998). In all species, FM1-43 labels synaptic vesicle clusters, and the loss of dye in response to nerve stimulation provides a sensitive measure of exocytosis.

Comparisons of FM1-43 loading and destaining of both ACh receptorless lines to wild-type control fish indicated that the developmental appearance of evoked release machinery did not depend on expression of AChRs. Indeed, the fraction and rate of destaining at FM1-43-labeled synaptic varicosities were comparable in wild type, nic-1, and sofa potato. The values obtained for zebrafish also compared favorably to the typical values published for amphibian and mammalian neuromuscular junctions (Betz and Bewick, 1992; Ribchester et al., 1994). Our observation that presynaptic exocytotic machinery can develop in the absence of postsynaptic receptors agrees with previously published studies showing that nerve-muscle synapses form in the presence of antagonists that block receptor function (Cohen, 1972; Ding et al., 1983; Landmesser and Szente, 1986). Our results are also consistent with the findings that dissociated spinal neuron somas and associated growth cones are capable of both spontaneous (Kidokoro and Yeh, 1982; Hume et al., 1983; Young and Poo, 1983; Chow and Poo, 1985) and evoked (Sun and Poo, 1987) transmitter release. However, studies using Xenopus have further indicated that responses to both spontaneous and stimulusevoked release of ACh from spinal neurons are potentiated on contact with muscle. Most notably, the frequency (Xie and Poo, 1986; Evers et al., 1989) and quantal size (Evers et al., 1989; Liou et al., 1997, 1999) of spontaneous events both increase soon after nerve-muscle contact in culture. The increase in quantal size was blocked when the cells were grown in D-tubocurarine to block ACh receptor activation, implicating a role of the receptors in the induction process (Liou et al., 1997, 1999).

Our results indicating that stimulus-evoked exocytosis is indistinguishable in ACh receptorless mutants and wild-type fish seemingly argue against receptor-mediated differentiation. One possibility is that synapse development in fish does not use the signaling pathway used by Xenopus. This explanation seems unlikely given the similarities between fish and frogs in all other aspects of synapse development. Another unlikely possibility is that we are studying very immature synapses and that early potentiation, reported for Xenopus, occurs subsequent to $3-5 \mathrm{~d}$ in fish. The initial contact between nerve and muscle occurs between 17 and $20 \mathrm{hr}$ after fertilization, and our recordings were performed between 96 and $120 \mathrm{hr}$ after fertilization. To obtain some quantitative measure of synaptic strength at this age, we recorded evoked synaptic current from 4-d-old wild-type fish and compared it with 14-d-old fish. In whole-cell recordings, we found no significant difference in mean evoked synaptic current amplitude between $14 \mathrm{~d}$ of age (mean $\pm \mathrm{SD} ; 3.5 \pm 2.0 \mathrm{nA} ; n=6$ fish) and $4 \mathrm{~d}$ of age ( $3.7 \pm 2.5 \mathrm{nA} ; n=12$ fish). Therefore, these

\footnotetext{
Figure 6. Destaining kinetics of FM1-43-loaded varicosities induced by electrical field stimulation. Representative time-lapse images of labeled varicosities (indicated by arrows) at $24 \mathrm{sec}$ intervals after the onset of electrical stimulation are shown for wild-type $(A)$, nic- $1(B)$, and sofa potato ( $C$ ) fish. Scale bars, $10 \mu \mathrm{m}$. The time course of fluorescence loss associated with the corresponding varicosities is shown in $A-C$. Time 0 corresponds to the onset of electrical stimulation. Fluorescence intensity was measured every $3 \mathrm{sec}$. The time required for decay from 90 to $10 \%$ of peak fluorescence determined for each trace is indicated alongside the corresponding legend bar. In several traces, the final plateau for destaining occurred beyond the time shown in the traces.
} 
Table 1. Comparisons of FM1- 43 labeling and destaining among wild-type, nic-1, and sofa potato fish

\begin{tabular}{|c|c|c|c|c|c|c|c|c|c|}
\hline & \multicolumn{3}{|c|}{ Initial labeling } & \multicolumn{3}{|c|}{ High potassium } & \multicolumn{3}{|c|}{ Electrical stimulation } \\
\hline & $\begin{array}{l}\text { Diameter } \\
(\mu \mathrm{m})\end{array}$ & $\begin{array}{l}\text { Intensity } \\
\text { (a.u.) }\end{array}$ & $n$ & $\begin{array}{l}\Delta F / F \\
(\%)\end{array}$ & $\begin{array}{l}T_{10-90 \%} \\
\text { (sec) }\end{array}$ & $n$ & $\begin{array}{l}\Delta F / F \\
(\%)\end{array}$ & $\begin{array}{l}T_{10-90 \%} \\
\text { (sec) }\end{array}$ & $n$ \\
\hline Wild type & $1.5 \pm 0.4$ & $560 \pm 80$ & 50 varicosities, 11 fish & $77 \pm 12$ & $47 \pm 22$ & 20 varicosities, 6 fish & $78 \pm 10$ & $94 \pm 32$ & 22 varicosities, 6 fish \\
\hline nic-1 & $1.5 \pm 0.3$ & $537 \pm 100$ & 45 varicosities, 11 fish & $74 \pm 13$ & $38 \pm 16$ & 18 varicosities, 5 fish & $72 \pm 10$ & $85 \pm 23$ & 18 varicosities, 5 fish \\
\hline sofa potato & $1.6 \pm 0.4$ & $560 \pm 97$ & 55 varicosities, 12 fish & $81 \pm 8$ & $43 \pm 16$ & 23 varicosities, 6 fish & $72 \pm 12$ & $80 \pm 30$ & 21 varicosities, 7 fish \\
\hline
\end{tabular}

All values represent mean \pm SD.

synapses in zebrafish are functionally well developed at the time corresponding to our FM1-43 measurements in zebrafish.

Our inability to identify differences between wild-type and receptorless mutants may be the result of limited resolution of FM1-43 destaining. Moreover, the potentiation in quantal size that results from activation of AChRs may go undetected by FM1-43 measurements of exocytosis, particularly should the potentiation result from either increased transmitter content per vesicle or closer apposition between nerve and muscle as proposed by Evers et al. (1989). Similarly, the associated increase in evoked response would also go undetected (Evers et al., 1989). However, our observation that increased receptor activation in wild-type fish leads to a higher rate of spontaneous destaining is in agreement with the studies from Xenopus showing increased frequency of miniature endplate currents after contact with muscle (Xie and Poo, 1986; Evers et al., 1989). This destaining was inhibited in wild-type fish by treatment with either $\alpha$-Btx or tetrodotoxin. We propose that the destaining in wild-type fish was not a consequence of synaptic transmission per se, because it was not observed in the mutant line relaxed. Relaxed fish are paralytic because of impaired excitation-contraction coupling (Ono et al., 2001), but the synaptic transmission appears normal when compared with wild-type fish. Instead, our collective findings suggest that the muscle contraction induced by synaptic responses may be responsible for the destaining. Consistent with this idea is the finding that neurotransmitter release is modulated by changes in muscle length. In frog motor neurons, the stretch-induced release represents a strong amplifier of the spinal stretch reflex (Chen and Grinnell, 1995, 1997). Stretch-induced release would be expected to be absent in paralytic fish and blocked by toxins that inhibit synaptic transmission.

The profound local differentiation of presynaptic terminals that is achieved for fully developed neuromuscular junctions points to the existence of strong signaling between nerve and muscle (Buchanan et al., 1989). The fact that we observed no obvious differences between wild-type and receptorless mutant fish precludes a central role of receptors in early establishment of presynaptic function. Such findings do not contraindicate the possible involvement of alternative signaling pathways that result from exocytosis from presynaptic terminals. Such signaling pathways could involve modulatory substances such as ATP (Hume and Honig, 1986), calcitonin gene-related peptide (Uchida et al., 1990), and other neuropeptides (Vilim et al., 1996) that are known to be coreleased at neuromuscular junctions. These could, in turn, lead to secretion of neurotrophins, thought to be causal to modulation of presynaptic function (Fu and Poo, 1991; Harish and Poo, 1992; Liou et al., 1997; Wang and Poo, 1997). Regardless of the trans-synaptic signal, our findings indicate that establishment of presynaptic calcium-dependent exocytotic machinery occurs independent of postsynaptic ACh receptors.

\section{References}

Betz WJ, Bewick GS (1992) Optical analysis of synaptic vesicle recycling at the frog neuromuscular junction. Science 255:200-203.
Betz WJ, Bewick GS (1993) Optical monitoring of transmitter release and synaptic vesicle recycling at the frog neuromuscular junction. J Physiol (Lond) 460:287-309.

Betz WJ, Mao F, Bewick GS (1992) Activity-dependent fluorescent staining and destaining of living vertebrate motor nerve terminals. J Neurosci 12:363-375.

Buchanan J, Sun YA, Poo MM (1989) Studies of nerve-muscle interactions in Xenopus cell culture: fine structure of early functional contacts. J Neurosci 9:1540-1554.

Chen BM, Grinnell AD (1995) Integrins and modulation of transmitter release from motor nerve terminals by stretch. Science 269:1578-1580.

Chen BM, Grinnell AD (1997) Kinetics, Ca dependence, and biophysical properties of integrin-mediated mechanical modulation of transmitter release from frog motor nerve terminals. J Neurosci 17:904-916.

Chow I, Poo MM (1985) Release of acetylcholine from embryonic neurons upon contact with muscle cell. J Neurosci 5:1076-1082.

Cohen MW (1972) The development of neuromuscular connexions in the presence of D-tubocurarine. Brain Res 41:457-463.

DiAntonio A, Petersen SA, Heckmann M, Goodman CS (1999) Glutamate receptor expression regulates quantal size and quantal content at the Drosophila neuromuscular junction. J Neurosci 19:3023-3032.

Ding R, Jansen JK, Laing NG, Tonnesen H (1983) The innervation of skeletal muscles in chickens curarized during early development. J Neurocytol 12:887-919.

Doi M, Iwasaki K (2002) Regulation of retrograde signaling at neuromuscular junctions by the novel C2 domain protein AEX-1. Neuron 33:249-259.

Evers J, Laser M, Sun YA, Xie ZP, Poo MM (1989) Studies of nerve-muscle interactions in Xenopus cell culture: analysis of early synaptic currents. J Neurosci 9:1523-1539.

Fu WM, Poo MM (1991) ATP potentiates spontaneous transmitter release at developing neuromuscular synapses. Neuron 6:837-843.

Gautam M, Noakes PG, Mudd J, Nichol M, Chu GC, Sanes JR, Merlie JP (1995) Failure of postsynaptic specialization to develop at neuromuscular junctions of rapsyn-deficient mice. Nature 377:232-236.

Granato M, van Eeden FJ, Schach U, Trowe T, Brand M, Furutani-Seiki M, Haffter P, Hammerschmidt M, Heisenberg CP, Jiang YJ, Kane DA, Kelsh RN, Mullins MC, Odenthal J, Nusslein-Volhard C (1996) Genes controlling and mediating locomotion behavior of the zebrafish embryo and larva. Development 123:399-413.

Harish OE, Poo MM (1992) Retrograde modulation at developing neuromuscular synapses: involvement of G-protein and arachidonic acid cascade. Neuron 9:1201-1209.

Hume RI, Honig MG (1986) Excitatory action of ATP on embryonic chick muscle. J Neurosci 6:681-690.

Hume RI, Role LW, Fischbach GD (1983) Acetylcholine release from growth cones detected with patches of acetylcholine receptor-rich membranes. Nature 305:632-634.

Kay AR, Alfonso A, Alford S, Cline HT, Holgado AM, Sakmann B, Snitsarev VA, Stricker TP, Takahashi M, Wu LG (1999) Imaging synaptic activity in intact brain and slices with FM1-43 in C. elegans, lamprey, and rat. Neuron 24:809-817.

Kidokoro Y, Yeh E (1982) Initial synaptic transmission at the growth cone in Xenopus nerve-muscle cultures. Proc Natl Acad Sci USA 79:6727-6731.

Kuromi H, Kidokoro Y (1998) Two distinct pools of synaptic vesicles in single presynaptic varicosities in a temperature-sensitive Drosophila mutant, shibire. Neuron 20:917-925.

Landmesser LT, Szente M (1986) Activation patterns of embryonic chick hind-limb muscles following blockade of activity and motor neurone cell death. J Physiol (Lond) 380:157-174.

Liou JC, Fu WM (1997) Regulation of quantal secretion from developing 
motor neurons by postsynaptic activity-dependent release of NT-3. J Neurosci 17:2459-2468.

Liou JC, Yang RS, Fu WM (1997) Regulation of quantal secretion by neurotrophic factors at developing motor neurons in Xenopus cell cultures. J Physiol (Lond) 503:129-139.

Liou JC, Chen YH, Fu WM (1999) Target-dependent regulation of acetylcholine secretion at developing motor neurons in Xenopus cell cultures. J Physiol (Lond) 517:721-730.

Nguyen QT, Son YJ, Sanes JR, Lichtman JW (2000) Nerve terminals form but fail to mature when postsynaptic differentiation is blocked: in vivo analysis using mammalian nerve-muscle chimeras. J Neurosci 20:6077-6086.

Ono F, Higashijima S, Shcherbatko A, Fetcho JR, Brehm P (2001) Paralytic zebrafish lacking acetylcholine receptors fail to localize rapsyn clusters to the synapse. J Neurosci 21:5439-5448.

Ono F, Shcherbatko A, Higashijima S, Mandel G, Brehm P (2002) The zebrafish motility mutant twitch once reveals new roles for rapsyn in synaptic function. J Neurosci 22:6491-6498.

Poyer JC, Zoran MJ (1996) Activity-dependent induction of functional secretory properties at cultured neuromuscular synapses of Helisoma. J Neurophysiol 76:2635-2643.

Ribchester RR, Mao F, Betz WJ (1994) Optical measurements of activitydependent membrane recycling in motor nerve terminals of mammalian skeletal muscle. Proc R Soc Lond B Biol Sci 255:61-66.

Sanes JR, Lichtman JW (2001) Induction, assembly, maturation and maintenance of a postsynaptic apparatus. Nat Rev Neurosci 2:791-805.
Sepich DS, Wegner J, O'Shea S, Westerfield M (1998) An altered intron inhibits synthesis of the acetylcholine receptor alpha-subunit in the paralyzed zebrafish mutant nic1. Genetics 148:361-372.

Sun YA, Poo MM (1987) Evoked release of acetylcholine from the growing embryonic neuron. Proc Natl Acad Sci USA 84:2540-2544.

Uchida S, Yamamoto H, Iio S, Matsumoto N, Wang XB, Yonehara N, Imai Y, Inoki R, Yoshida H (1990) Release of calcitonin gene-related peptidelike immunoreactive substance from neuromuscular junction by nerve excitation and its action on striated muscle. J Neurochem 54:1000-1003.

Vilim FS, Price DA, Lesser W, Kupfermann I, Weiss KR (1996) Costorage and corelease of modulatory peptide cotransmitters with partially antagonistic actions on the accessory radula closer muscle of Aplysia californica. J Neurosci 16:8092-8104.

Wan J, Poo M (1999) Activity-induced potentiation of developing neuromuscular synapses. Science 285:1725-1728.

Wang XH, Poo MM (1997) Potentiation of developing synapses by postsynaptic release of neurotrophin-4. Neuron 19:825-835.

Westerfield M, Liu DW, Kimmel CB, Walker C (1990) Pathfinding and synapse formation in a zebrafish mutant lacking functional acetylcholine receptors. Neuron 4:867-874.

Xie ZP, Poo MM (1986) Initial events in the formation of neuromuscular synapse: rapid induction of acetylcholine release from embryonic neuron. Proc Natl Acad Sci USA 83:7069-7073.

Young SH, Poo MM (1983) Spontaneous release of transmitter from growth cones of embryonic neurones. Nature 305:634-637. 